\title{
Les Ptéridophytes du Maroc : richesse, diversité et état de conservation
}

\author{
Mohamed Fennane \& Mohamed Ibn Tattou \\ Université Mohammed V de Rabat, Institut Scientifique, \\ Avenue Ibn Battouta, Agdal, 10106, Rabat, Maroc.
}

\section{Correspondence}

M. Fennane

e-mail : fennane@israbat.ac.ma

Reçu: 5 junio 2017

Accepté: 5 septiembre 2017

Publié on line: diciembre 2017

\begin{abstract}
Résumé
La flore ptéridophytique du Maroc compte une soixantaine d'espèces, réparties entre 25 genres et 17 familles. Les auteurs présentent cette flore sur les plans quantitatif et qualitatif en faisant des comparaisons avec des pays voisins et avec l'ensemble de la région Euro-Méditerranéenne. Les traits écologiques remarquables des fougères du Maroc sont soulignés; des exemples précis sont donnés. Une clé dichotomique aidant à l'identification des familles, des genres et des espèces est fournie. Les états de présence et de conservation des espèces sont exposés et discutés. Une attention toute particulière est accordée à 16 espèces, considérées rares ou menacées, et à 22 autres pour lesquelles les informations sont insuffisantes. Les auteurs concluent qu'il y a urgence pour entreprendre des actions de protection de certaines espèces qui sont sérieusement menacées.
\end{abstract}

Mots clés: Pteridophyta, Maroc, écologie, chorologie, conservation.

\section{Resumen}

La flora pteridofítica de Marruecos cuenta con una sesentena de especies, repartidos en 25 géneros y 17 familias. Se presenta esta flora en términos cuantitativos y cualitativos, realizando una comparación con los países vecinos y con el conjunto de la región Euro-Mediterránea. Se señalan los caracteres ecológicos más interesantes a los helechos de Marruecos, ofreciéndose algunos ejemplos concretos. Se añade una clave dicotómica para la identificación de familias, géneros y especies. Se discute la situación, la presencia y conservación de las especies. Una atención particular merecen 16 especies consideradas como raras o amenazadas y 22 especies para las cuales la información es insuficiente. Se concluye que es urgente emprender acciones de protección de ciertas especies que están seriamente amenazadas.

Palabras clave: Pteridophyta, Marruecos, ecología, corología, conservación.

\section{Introduction}

Avec une soixantaine d'espèces, les ptéridophytes au Maroc, représentent environ $1,5 \%$ de la flore vasculaire nationale. Cette présence importante et significative n'est pas appréciée à sa juste valeur par les non-botanistes, y compris les gestionnaires de la biodiversité. Et pour cause, il y aurait d'une part le fait que nos fougères n'ont pas de valeur socio-économique notable, du moins jusqu'à présent, et d'autre part, elles n'impressionnent pas les paysages naturels, sauf de rares exceptions ; presque toutes sont des hémicryptophyes ou des géophytes à rhizome et non des arbres ou des arbustes.

Sur le plan des recherches et des connaissances, 
un nombre important d'espèces posent des problèmes taxonomiques et nomenclaturaux. II y a aussi un grand manque d'informations sur les aires de répartition géographiques.

Dans ce travail, il est question de donner un état des lieux pour aider les chercheurs à mieux orienter leurs investigations et les gestionnaires à définir les priorités et choisir les espèces cibles pour des opérations de protection et/ou de conservation.

\section{Richesse et diversité des ptéridophytes au Maroc}

Les données bibliographiques et webographiques récentes (Fennane \& Ibn Tattou 2005 ; Dobignard \& Chatelain 2010 ; EMBase ; PPGI 2016) sur les cryptogames vasculaires (fougères ou Ptéridophytes) au Maroc révèlent l'existence de 66 espèces, dont 7 de présence douteuse (Asplenium anceps Hook. \& Grev., Asplenium foreziense Magnier, Dryopteris borren (Newman) Oberh. \& Tavel, Equisetum arvense L., Polypodium interjectum Shivas, Allosorus tinaei (Tod.) Christenh., Azolla cristata Kaulf.) et 1 naturalisée (Azolla filiculoides Lam.) ; toutes appartenant à 25 genres et 17 familles. Les familles sont comprises ici selon la nouvelle classification phylogénétique (PPGI 2016). A l'échelle nationale, ces chiffres paraissent faibles par rapport à l'ensemble de la flore vasculaire du pays qui s'élève à 155 familles, 1005 genres (dont 24 de présence douteuse) et 4248 espèces (dont 261 de présence douteuse et 74 naturalisées) (Fennane \& Ibn Tattou 2012). Mais, cette présence s'avère remarquable quand on fait des comparaisons avec des pays voisins comme l'Algérie et l'Espagne et avec l'ensemble de l'aire Euro-Méditerranéenne (Fig. 1). Les richesses du Maroc et de l'Algérie sont très voisines, assez loin derrière l'Espagne.

L'examen de la composition de la flore

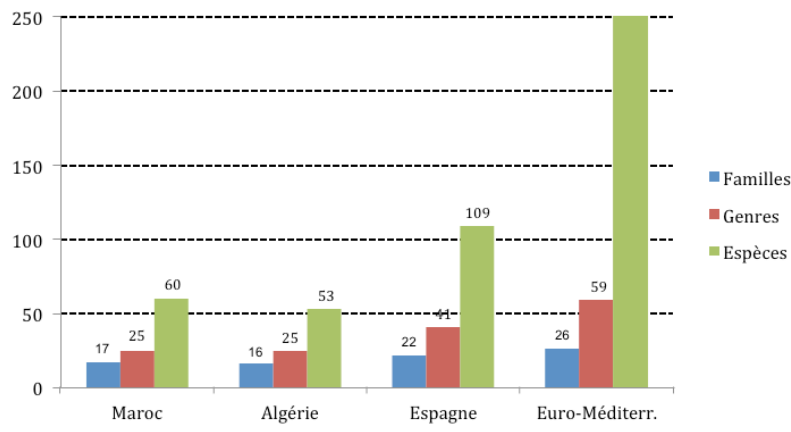

Figure 1. Nombres de familles, genres et espèces de Ptéridophytes au Maroc et dans des pays voisins ptéridophytique du Maroc et de pays voisins laisse montrer une grande diversité (Tab. 1). Toutes les familles sont représentées chez nous par un ou deux genres seulement, sauf les Ptéridacées. La même situation se retrouve en Algérie et un peu moins en Espagne. Les genres les plus riches sont Asplenium (18 espèces au Maroc et 38 dans l'aire Euro-Méditerranéenne) et Isoëtes (respectivement 6 et 21 espèces) ; tous les autres ne comptent pas plus de deux espèces chacun, sauf Allosorus (5 espèces), Ophioglossum (3 espèces) et Polystichum (3 espèces).

Sur le plan de l'endémisme, la flore ptéridophytique du Maroc ne montre pas beaucoup d'originalités ; on note une seule espèce endémique nationale, Selaginella balansae (A. Braun) Hieron., et 4 autres à des échelles plus larges: Asplenium anceps Hook. \& Grev. (Maroc, Algérie et îles Macaronésiennes), Asplenium hispanicum (Coss.) Greuter \& Burdet (Maroc, Algérie et Espagne), Davallia canariensis (L.) Sm. (Maroc, Péninsule Ibérique et îles Macaronésiennes) et Asplenium hemionitis L. (Maroc, Algérie, Péninsule Ibérique et îles Macaroné-siennes).

\section{Ecologie et chorologie}

D'une manière générale, les fougères sont réputées être liées aux milieux humides, ombragés. Au Maroc, ce fait s'exprime clairement par leur répartition; elles sont plus présentes dans les régions montagneuses bien arrosées (Fig. 2 et 3). La chaîne Rifaine est la plus riche, avec pas moins de 48 espèces, dont Pteris incompleta Cav. et Paragymnopteris marantae (L.) K.H. Shing, observées uniquement dans le Rif occidental.

Les zones les plus chaudes et arides du pays (Ms et As) ne montrent pas plus de sept espèces, dont Equisetum ramosissimum Desf., cosmopolite, présente un peu partout sur les sols humides, entre 0 et $2000 \mathrm{~m}$. Parmi les autres espèces, quatre s'observent dans plusieurs autres régions, à savoir Asplenium ceterach L., Allosorus acrostichus (Balb.) Christenh., Allosorus pteridioides (Reichard) Christenh. et Cosentinia vellea (Aiton) Tod.. Finalement, seules Ophioglossum polyphyllum A. Braun et Davallia canariensis (L.) Sm. sont intéressantes à souligner : la première est connue uniquement dans le Nord du Sahara atlantique ; la deuxième montre une aire très disloquée divisée en deux grands sous-ensembles, un méridional et un septentrional. Nous reviendrons plus loin en détail sur ces aires de répartition. Par ailleurs, ces deux espèces sont aussi d'une grande valeur biogéographique. Ophioglossum est chez nous à la limite nord-occidentale de son aire mondiale 


\begin{tabular}{|c|c|c|c|c|c|c|c|c|}
\hline \multirow[b]{2}{*}{ Familles } & \multicolumn{2}{|c|}{ Maroc } & \multicolumn{2}{|c|}{ Algérie } & \multicolumn{2}{|c|}{ Espagne } & \multicolumn{2}{|c|}{$\begin{array}{l}\text { Région Euro- } \\
\text { Méditerranéenne }\end{array}$} \\
\hline & G & SP & G & SP & G & SP & G & SP \\
\hline Aspleniaceae & 1 & 18 & 1 & 13 & 1 & 16 & 1 & 38 \\
\hline Athyriaceae & 1 & 1 & 1 & 1 & 2 & 3 & 3 & 6 \\
\hline Blechnaceae & 1 & 1 & 2 & 2 & 2 & 2 & 2 & 8 \\
\hline Cystopteridaceae & 1 & 1 & 2 & 2 & 2 & 4 & 2 & 6 \\
\hline Davalliaceae & 1 & 1 & 0 & 0 & 1 & 1 & 1 & 1 \\
\hline Dennstaedtiaceae & 1 & 1 & 1 & 1 & 1 & 1 & 3 & 4 \\
\hline Dryopteridaceae & 2 & 5 & 2 & 4 & 2 & 17 & 5 & 43 \\
\hline Equisetaceae & 1 & 2 & 1 & 2 & 1 & 8 & 1 & 10 \\
\hline Isoëtaceae & 1 & 6 & 1 & 5 & 1 & 9 & 1 & 21 \\
\hline Marsileaceae & 2 & 3 & 2 & 4 & 2 & 4 & 2 & 9 \\
\hline Ophioglossaceae & 2 & 4 & 1 & 2 & 2 & 5 & 2 & 12 \\
\hline Osmundaceae & 1 & 1 & 1 & 1 & 1 & 1 & 1 & 1 \\
\hline Polypodiaceae & 1 & 2 & 1 & 1 & 1 & 3 & 5 & 8 \\
\hline Pteridaceae & 6 & 10 & 6 & 11 & 8 & 14 & 11 & 33 \\
\hline Salviniaceae & 1 & 1 & 1 & 1 & 2 & 2 & 2 & 7 \\
\hline Selaginellaceae & 1 & 2 & 1 & 1 & 1 & 3 & 1 & 6 \\
\hline Thelypteridaceae & 1 & 2 & 1 & 2 & 3 & 5 & 3 & 6 \\
\hline Culcitaceae & 0 & 0 & 0 & 0 & 1 & 1 & 1 & 1 \\
\hline Hymenopyllaceae & 0 & 0 & 0 & 0 & 2 & 2 & 2 & 4 \\
\hline Lycopodiaceae & 0 & 0 & 0 & 0 & 3 & 4 & 3 & 14 \\
\hline Psilotaceae & 0 & 0 & 0 & 0 & 1 & 1 & 1 & 1 \\
\hline Woodsiaceae & 0 & 0 & 0 & 0 & 1 & 3 & 1 & 5 \\
\hline Cyatheaceae & 0 & 0 & 0 & 0 & 0 & 0 & 2 & 3 \\
\hline Dicksoniaceae & 0 & 0 & 0 & 0 & 0 & 0 & 1 & 1 \\
\hline Nephrolepidaceae & 0 & 0 & 0 & 0 & 0 & 0 & 1 & 2 \\
\hline Onocleaceae & 0 & 0 & 0 & 0 & 0 & 0 & 1 & 2 \\
\hline Total & 25 & 60 & 25 & 53 & 41 & 109 & 59 & 252 \\
\hline
\end{tabular}

Tableau 1. Richesse et diversité des Ptéridophytes au Maroc, comparées à des pays voisins. (Références : Fennane \& Ibn Tattou 2005, Dobignard \& Chatelain 2010, EMBase) 


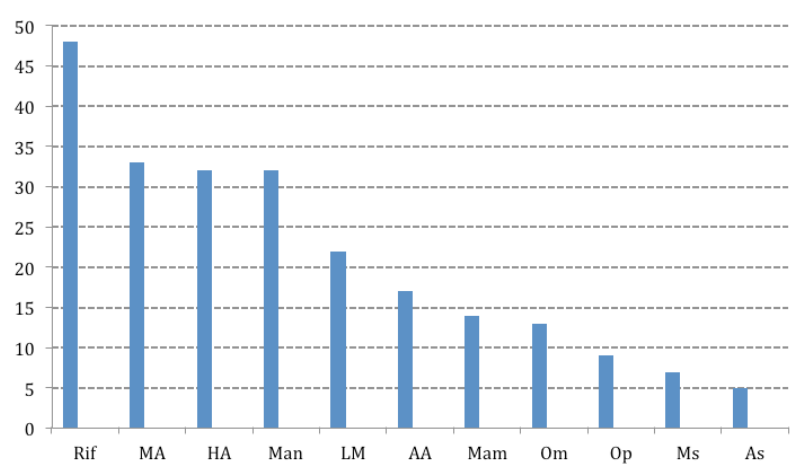

Figure 2. Nombres d'espèces de fougères par région au Maroc (abréviations : voir Fig. 3).

qui concerne les îles Macaronésiennes, l'Afrique, l'Arabie et l'Australie. En revanche, Davallia, comme nous venons de le voir, est une eury-endémique des îles Macaronésiennes, du Maroc et de la Péninsule Ibérique.

Sur le plan écologique, la majorité des fougères s'observent sur des substrats humides : fissures des rochers, talus, vieux murs, troncs d'arbres etc. Certaines sont aquatiques à sub-aquatiques ; c'est le cas notamment pour Azolla, Isoëtes, Marsilea et Pilularia. Les espèces pouvant supporter des conditions relativement sèches sont peu nombreuses, parmi elles: Asplenium ceterach, Ophioglossum polyphyllum, Cosentinia vellea, Allosorus hispanicus, Allosorus pteridioides... Enfin, il convient de signaler des cas bien particuliers, comme Asplenium marinum L., lié aux grottes et falaises maritimes ou sublittorales et Botrychium lunaria (L.) Sw., spécial aux pozzines de hautes montagnes.

\section{Clés d'identification}

\section{Clé des familles}

1 Tiges articulées. Feuilles réduites, verticillées et soudées sous forme d'une gaine autour des tiges. . Equisetaceae

1' Tiges non articulées. Feuilles développées ............2

2 Plante aquatique flottante ....................Salviniaceae

2' Plante aquatique ou terrestre, enracinée................ 3

3 Feuilles sans pétiole différencié.............................. 4

3' Feuilles à pétiole bien différencié, court ou long.....6 6

4 Plante à tige réduite sous forme de bulbe («corm»). Feuilles linéaires, toutes en rosette basale..... Isoëtaceae

4' Plante sans bulbe. Feuilles filiformes ou ovalestriangulaires, non en rosette basale.

5 Plante aquatique. Feuilles filiformes .

...Marsileaceae

5' Plante terrestre. Feuilles semblables et spiralées, ou dimorphes et disposées sur un même plan sur 4 rangées ; ligule présente. Selaginellaceae

6 Feuilles à 4 folioles, longuement pétiolées

Marsileaceae

6' Feuilles simples ou divisées, mais non 4-foliolées .7

7 Sporanges en épis ou en panicules, sans anneau ou à anneau rudimentaire.

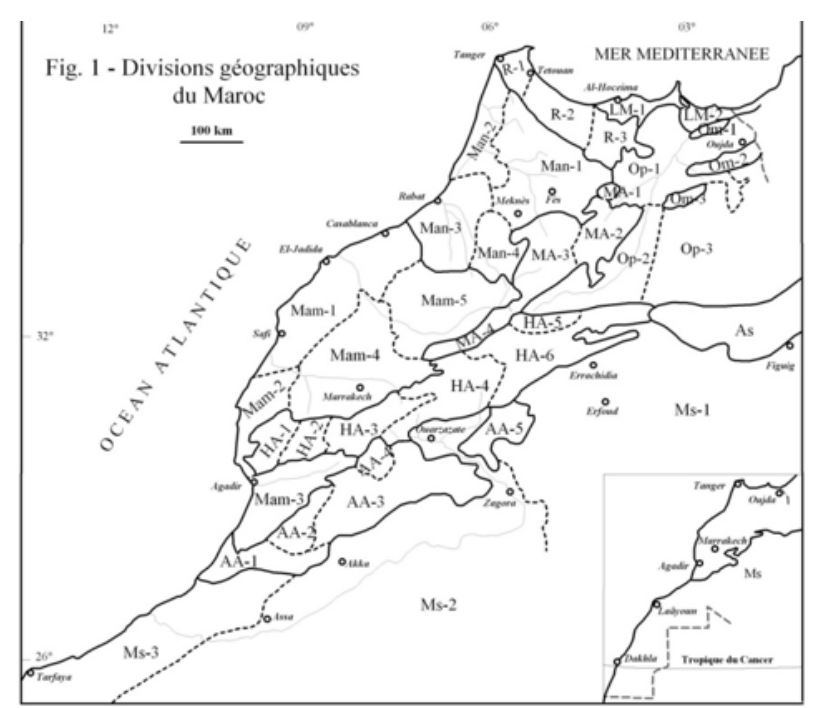

Figure 3. Divisions géographiques du Maroc (Ms : Maroc saharien, As : Atlas Saharien, AA : Anti-Atlas, HA: Haut Atlas, MA : Moyen Atlas, Mam : Maroc atlantique moyen, Man : Maroc atlantique nord, Op : hauts plateaux de l'oriental, Om: monts de l'Oriental, LM : Littoral Méditerranéen, R : Rif)

7' Sporanges disposées sur la face inférieure des feuilles, à anneau nettement différencié ................ 9

8 Panicules de sporanges portées par le tiers supérieur de la feuille (= fronde); anneau central rudimentaire. Plante dépassant $40 \mathrm{~cm}$ de long........Osmundaceae

8' Epis ou panicules de sporanges disposés sur les divisions latérales des feuilles, anneau absent. Plante de moins de $40 \mathrm{~cm}$............ Ophioglossaceae

9 Plante annuelle, prothalle se multipliant par bourgeonnement, \pm pérennant dans les stations à humidité permanente. Limbe foliaire très mince, très polymorphe, arrondi-réniforme et \pm digité dans les feuilles inférieures, ovale à oblong-lancéolé dans les suivantes, où il est 1-3 pinnatiséqué, obtus ; marges planes, ne recouvrant pas les sores .......Pteridaceae (Anogramma)

9' Plante nettement vivace, à rhizome \pm développé. Autres caractères non réunis ...............................10

10 Feuilles dimorphes, les fertiles différentes des stériles, 1-pinnatiséquées. .................. Blechnaceae

10' Feuilles homomorphes........................................ 11

11 Feuilles à limbe entier, lobé, palmé ou dichotome.... Aspleniaceae p.p.

11' Feuilles à limbe pinnatipartite à 1-4-pinnatiséqué 12

12 Limbe foliaire pinnatipartite................................. 13

12 ' Limbe foliaire bipinnatipartite ou 1-4-pinnatiséqué ...

$\ldots 14$

13 Feuilles à limbe glabre; sores nues .

Polypodiaceae p.p.

13' Face inférieure des feuilles couverte d'écailles; sores à indusie latérale..... Aspleniaceae p.p.

14 Marges du limbe révolutées au moins à maturité 15

14' Marges du limbe \pm planes.................................. 17

15 Sores sans indusie, couvertes seulement par la marge du limbe foliaire...........................Pteridaceae

15' Sores à indusie ciliée ou poilue ............................. 16 
16 Limbe foliaire 2-3-pinnatiséqué, largement ovaletriangulaire................................. Dennstaedtiaceae

16' Limbe foliaire pinnatiséqué, lancéolé à oblonglancéolé, à face inférieure poilue.. Thelypteridaceae

17 Feuilles distiques le long du rhizome ; pétiole articulé à la base; sores apicales, submarginales disposées à l'extrémité des nervures; indusie en coupe.

Davalliaceae

17' Feuilles fasciculées. Autres caractères non réunis18

18 Sores arrondies ou elliptiques, disposées sur deux rangées, de part et d'autre de la nervure principale des divisions primaires ; limbe 1-pinnatipartite à 1-pinnatiséqué; sporanges longuement pédicellés...

Polypodiaceae

18'-Sores non disposées comme ci-dessus; autres caractères non réunis .......................................... 19

19 Pennes courtement pétiolulées.............................20

19 Pennes sessiles ....................................................21

20 Limbe 1-2-pinnatiséqué; pennes 7-18 de chaque côté, les inférieures opposées, les supérieures alternes; feuilles longues $10-50 \mathrm{~cm}$

Cystopteridaceae

20' Limbe 2-3-pinnatiséqué; pennes jusqu'à 40 de chaque côté, alternes; feuilles longues (20-)30(-150) $\mathrm{cm}$; sores oblongues, arquées; indusie virguliforme ou réniforme, persistante...................... Athyriaceae

21 Face inférieure au moins du limbe poilue; indusie ciliée ou poilue Thelypteridaceae

21' Limbe glabre; indusie glabre si présente ..............22

22 Sores elliptiques, oblongues ou linéaires; indusie absente ou latérale Aspleniaceae

22 ' Sores orbiculaires; indusie orbiculaire à réniforme ou en fer à cheval, peltée ou non.........Dryopteridaceae

\section{Clés des genres et des espèces}

\section{Aspleniaceae : Au Maroc, un seul genre Asplenium}

1 Feuille à limbe coriace, pinnatiséqué, couvert sur la face inférieure de squamules brunâtres, brillantes, saillantes à la à face supérieure verte et glabre; rachis squamuleux.

A. ceterach

1' Face inférieure des feuilles non couvertes d'écailles 2

2 Limbe entier cordiforme à 3-5-lobé, pourvu à la base de 2 lobes latéraux entiers ou \pm bilobés et au-dessus d'un lobe médian plus long; lobe médian \pm acuminé; lobes latéraux obtus ou acuminés, horizontaux ou ascendants, présentant souvent vers la base un lobule arrondi, rarement aigu, défléchi, à 5 nervures primaires palmées avec de nombreuses nervures secondaires pennées souvent une ou plusieurs fois dichotomes; marge ondulée-subcrénelée.

A. hemionitis

2' Limbe non comme ci-dessus.

3

3 Limbe simple, à base cordiforme ........................... 4

3' Limbe diversement divisé, ou si entier à base non cordiforme, ni hastée

4 Feuilles des jeunes pieds à limbe ovale, obtus au sommet; celles des pieds adultes à limbe hasté, un peu rétréci et oblong-lancéolé au-dessus des oreillettes basilaires souvent divariquées et aiguës, parfois obtuses et peu saillantes, rarement lobées. Nervures secondaires plus espacées, ramifiées en majeure partie loin de leur base, non épaissies au sommet. Sores oblongs, ne dépassant guère 13 $\mathrm{mm}$........................ A. sagittatum subsp. glabrum

4' Limbe linéaire-lancéolé, obtus ou aigu et même un peu acuminé, à marge entière, à face inférieure un peu squamuleuse, surtout sur le rachis, bientôt glabre. Sores linéaires inégaux, les plus longs pouvant atteindre $30 \mathrm{~mm}$............A. scolopendrium

5 Limbe trifide à triséqué ou inégalement dichotome. Pétiole vert sauf à la base brun-rouge.................... 6

5' Limbe 1-4-penné.................................................. 7

6 Indusie à marge \pm érodée. Limbe étalé, parfois même réfléchi, coriace, court, trifide ou triséqué ; segments cunéi-formes à la hase, rhomboïdaux-oblongs, irrégulièrement laciniés ou dentés, couverts de poils glanduleux.............................................. A. seelosii

6' Indusie à marge entière. Limbe inégalement dichotome, parfois trifide, parfois entier, coriace, glabre; segments linéaires-cunéiformes, le plus souvent élargis et dentés ou laciniés au sommet (dents dressées linéaires-lancéolées aiguës....

A. septentrionale

7 Limbe 1-penné ........................................................ 8

7' Limbe 2-4-penné.................................................. 15

8 Rachis ailé. Rhizome court ................................... 9

8' Rachis non ailé ................................................... 11

9 Rachis \pm noir ou vert, étroitement ailé par la décurrence des pinnules. Feuilles en touffes lâches.

A. marinum

9' Rachis ailé sur toute sa longueur. Feuilles en touffes denses ...................................................... 10

10 Rachis très épais, rougeâtre brillant, ailé sur toute sa longueur, 2 ailes sur la face supérieure, 1 aile sur la face inférieure.....

A. anceps

10' Pétiole brun-sombre ...................... A. trichomanes

a Pennes lancéolées à oblongues-lancéolées, symétriques, à marges incisées-crénelées; les inférieures nettement hastées.... subsp. pachyrachis

a' Pennes suborbiculaires, ovales ou oblongues, asymétriques; à marges entières ou parfois dentéecrénelée ............................... subsp. quadrivalens

11 Pétiole plus long que le limbe ......... A. ruta-muraria

11' Pétiole plus court que le limbe ou l'égalant............12

12 Plante couverte de poils glanduleux \pm denses. Limbe oblong-lancéolé ; pennes ovales-anguleux.

A. petrarchae p.p.

12' Plante glabre ou à rachis seulement densément villeux .............................................................. 13

13 Pétiole densément villeux à longs poils en mélange avec des poils glanduleux courts et vers la base de grosses glandes subsessiles...

A. hispanicum

13' Pétiole glabre ou légèrement écailleux et devenant glabrescent

14 Pétiole brun-rouge brillant................... A. obovatum

a Pinnules à dents aiguës subspinuleuses .................. ..................................................... subsp. billotii

a' Pinnules arrondies, à dents courtes, obtuses subsp. obovatum

14' Pétiole brun-pourpre à sa base seulement, vert ailleurs...... A. viride 
15 Sores courtement elliptiques.

A. petrarchae

15 ' Sores linéaires à oblongues. 16

16 Pétiole de couleur verte sauf à la base brun-rouge. Limbe des jeune feuilles lâchement squamuleux et à glandes \pm pédicellées, bientôt glabrescent et à la fin glabre.

A. ruta-muraria

16' Pétiole brun, noirâtre, pourpre-noir à noir ............. 17

17 Pennes à nervation flabellée, plusieurs fois dichotome. Pétiole à écailles noires piliformes.........

A. aethiopicum

17' Nervation des pennes non flabelliformes. Pétiole glabre. 18

18 Pétiole égalant ou plus long que le limbe

A. adiantum-nigrum

18' Pétiole plus court de le limbe .. 19

19 Sores insérées vers la base des nervures de la face inférieure.

A. fontanum

19' Sores insérées sur les nervures dans la partie médiane de la face inférieure......

A. foreziense

Athyriaceae : Au Maroc, une seule espèce Athyrium filix-femina

Blechnaceae : Au Maroc, une seule espèce Blechnum spicant

Cystopteridaceae : Au Maroc, une seule espèce Cystopteris fragilis

a Spores rugueuses à surface granuleuse ..................

.....................................................subsp. dickieana

a' Spores à surface épineuse .................................. b

b Surface des spores à épines en lames plus ou moins découpées à leur sommet..............subsp. diaphana

b' Surface des spores à épines coniques ................... c

c limbe 3-penné; divisions ultimes larges jusqu'à $1,5 \mathrm{~mm}$, linéaires-rectangulaires, à sommet généralement émarginé; nervures se terminant dans les émarginations................................subsp. alpina

c' Limbe 2(3)-penné; divisions ultimes de plus de $2 \mathrm{~mm}$ de large, \pm largement ovales-oblongues, à marges dentées, à dents aiguës ou obtuses, rarement émarginées, nervures se terminant dans les émarginations ou au sommet des dents subsp. fragilis

Davalliaceae: Au Maroc, une seule espèce Davallia canariensis

Dennstaedtiaceae: $\mathrm{Au}$ Maroc, une seule espèce Pteridium aquilinum

\section{Dryopteridacae}

1 Limbe foliaire largement elliptique, 1-2-pinnatiséqué; pinnules symétriques. Indusie réniforme Dryopteris

1' Limbe foliaire lancéolé, 1-3-pinnatiséqué; segments ultimes \pm falciformes, auriculées à la base et asymétriques, à lobule terminal plus développé. Indusie peltée

Polystichum

\section{Dryopteris}

1 -Pétiole densément paléacé seulement à sa base; écailles à majorité lancéolées, brun-clair à brun- rougeâtre. Limbe largement elliptique. Pinnules à bords légèrement courbés et convergents au sommet; lobules terminaux aigus ou obtus.

D. filix-mas

1' Pétiole et rachis densément paléacés; écailles à majorité filiformes, brun-sombre au moins à leur base. Limbe lancéolé à triangulaire-lancéolé. Pinnules à bords parallèles, entiers ou lobulés.......2

2 Pinnules lobées, à lobules rectangulaires $\boldsymbol{D}$. borreri

2' Pinnules entières à non nettement lobées. Zone apicale des pinnules à dents aiguës, peu nombreuses

D. affinis

\section{Polystichum}

1 Limbe 1-penné...................................... Ionchitis

1' Limbe 2(3) penné................................................ 2

2 Pinnules presque toutes pétiolulées, non décurrentes........... P. setiferum subsp. aculeatum

2' Pinnules inf. seules pétiolulées, les autres sessiles et \pm connées avec le rachis. ........P. aculeatum subsp. lobata

Equisetaceae : Au Maroc, un seul genre Equisetum

1 Tiges stériles blanches ; rameaux verts, les fertiles simples. Epi gros, conique, à sommet obtus. Stomates superficiels...........................E. telmateia

1' Tiges toutes semblables, vertes, ordinairement très rameuses. Epi court, ovoïde et apiculé. Stomates enfoncés dans l'épiderme.

E. ramosissimum

Equisetum arvense L. : Présence jamais confirmée au Maroc ; confusion avec E. ramosissimum.

Equisetum giganteum L. : Cité par l'IUCN [http:// www.iucnredlist.org] (accès, avril 2017) ; indication erronée.

Isoetaceae: Au Maroc, un seul genre Isoetes

1 Phyllopodes généralement absentes. Sporange couvert partiellement ou totalement le voile. Plante aquatique longtemps submergée........................... 2

1' Phyllopodes entourant la tige bulbiforme. Sporange totalement couvert par le voile. Plante terrestre ou aquatique, mais jamais submergée

2 Voile totalement absent.......................... setacea

2' Voile présent, parfois réduit en arc de cercle...........3

3 Rhizome couvert d'écailles stériles......... I. tiguliana

3' Rhizome sans écailles .......................................... 4

4 Feuilles longues de $5-30 \mathrm{~cm}$...................... velata

4' Feuilles longues de $40-80 \mathrm{~cm}$............. I. Iongissima

a Voile couvrant la totalité du sporange............ subsp. longissima

a' Voile ne couvrant pas la totalité du sporange .......... b

b Voile en arc réduit ..........................subsp. adspersa

b' Voile couvrant au moins le quart du sporange...........

.................................................. subsp. intermedia

5 Phyllopode à 2 dents latérales en «cornes» longues 3-10 mm, la centrale réduite ou absente ... I. histrix

5' Phyllopode à 3 dents réduites égales ou inégales ...

.. l. durieui

\section{Marsileaceae}

1 Feuilles cylindriques subulées, sessiles 
Pilularia minuta

1' Feuilles à 4 folioles, longuement pétioléesMarsilea.

2 Feuilles glabres. Rhizome grêle très allongé, rampant, radicant, poilu puis glabrescent ; sporocarpes sur des pédicelles 2 fois plus longs qu'eux, insérés par 2-3 vers la base du pétiole ou plus haut, ellipsoïdescomprimés ou lenticulaires, arrondis au sommet, \pm tronqués et portant 2 dents inégales (la supérieure plus longue) à la base, couverts dans la jeunesse de poils articulés apprimés, puis glabres, non marginés, lisses. Sores 5-6 de chaque côté............. M. minuta

2' Folioles cunéiformes \pm pubescentes. Rhizome épais, émettant des stolons allongés et grêles, pubescent ; sporocarpes disposés en 2 rangs sur le rhizome, insérés solitairement sur la base des feuilles, portés par un pédicelle égalant au plus le 1/4 du sporocarpe, lenticulaires couverts de poils articulés 3-8-cellulaires, à 2 dents rapprochées très courtes, parfois presque nulles. Sores 3-4 de chaque côté....

M. strigosa

\section{Ophioglossaceae}

1 Feuille à segment stérile pinnatiséqué ; segment fertile ramifié paniculé Botrychium lunaria

1' Feuille à segment stérile entier, lancéolé ; segment fertile spiciforme, avec 2 rangées de sporanges.. Ophioglossum..

2 Appendice sporangifère naissant le plus souvent au-dessous du milieu du sporophylle. Rhizome \pm allongé, un peu épaissi, à ramifications grêles traçantes, nu, à racines fasciculées, peu serrées, brunes. Limbe étroitement lancéolé, longuement atténué en pétiolule à sa base, brièvement atténué et obtusiuscule au sommet

O. Iusitanicum

2' Appendice sporangifère naissant vers le milieu ou au au-dessus du milieu du sporophylle. Rhizome court

3 - Plante haute de $3-6 \mathrm{~cm}$. Epi des sporanges plus ou moins longuement pédonculé, plus court que le limbe (dans les spécimens encore immatures), linéaire, mucroné au sommet. Limbe largement lancéolé, atténué à la base, non pétiolée, \pm aigu et nettement apiculé au sommet.....

O. polyphyllum

3'- Plante haute de $10-35 \mathrm{~cm}$. Epi des sporanges plus ou moins longuement pédonculé, dépassant ordinairement le limbe, linéaire, mucroné au sommet. Limbe obtus, rarement acutiuscule et mucroné au sommet, plus ou moins brusquement atténué à la base, non pétiolée.

$$
\text { O. vulgatum }
$$

\section{Osmundaceae: Au Maroc une seule espèce Osmunda regalis}

Polypodiaceae: Au Maroc, un seul genre Polypodium

1 Sores à paraphyses longues de 200-1800 $\mu \mathrm{m}$.......... P. cambricum

1' Sores sans paraphyses, parfois à poils glanduleux de 3-4 cellules atteignant $140 \mu \mathrm{m}$..

2 Ecailles du rhizome longues de $3-4 \mathrm{~mm}$. Limbe ovalelancéolé à largement triangulaire. Anneau brunrougeâtre, formé de (7)11-14(18) cellules; cellules basales de l'anneau (0)1(2 .................. P. vulgare

2' - Ecailles du rhizome longues de 4-6 mm. Limbe ovale-lancéolé à lancéolé. Anneau de couleur brune à marron jaunâtre, formé de (4)7-10(13) cellules; cellules basales de l'anneau (1)2-3(4 P. interjectum

\section{Pteridaceae}

1 -Plante annuelle ou pérennante des milieux constamment humides; prothalle se multipliant par bourgeonnement; limbe très mince, très polymorphe, arrondi-réniforme et \pm digité dans les feuilles inf., ovale à oblong-lancéolé dans les suivantes, où il est 1-3 pinnatiséqué, obtus. Marges planes, ne recouvrant pas les sores. . Anogramma leptophylla

1' Plante à rhizome \pm développé, généralement paléacé; feuilles à limbe 1-4-pinnatiséqué..............2

2 Limbe densément villeux et rubigineux dessous, lâchement villeux et vert dessus, lancéolé, 1-3-pinnatiséqué; sores cachées par les poils..........

Cosentinia vellea

2' Limbe glabre ou à face inférieure couverte d'écailles éparses ou de poils glanduleux; sores habituellement couvertes par la marge révolutée du limbe............. 3

3 Pétiole couvert abondamment d'écailles ferrugineuses linéaires- lancéolées.

Paragymnopteris marantae

3' Pétiole non couvert d'écailles ferrugineuses........... 4

4 Pinnules pétiolulées, divisions ultimes flabelliformes et irrégulièrement lobées au sommet dans les sporophylles, obovales-cunéiformes, \pm inciséesserrulées dans les feuilles stériles; nervures fines, plusieurs fois dichotomes, atteignant le bord du limbe au sommet des lobes et dents. Lobules marginaux sorifères carrés à réniformes, bruns.

Adiantum capillus-veneris

4' Pinnules sessiles, subsessiles ou décurrentes; autres caractères non réunis

5 Pennes sessiles chez les feuilles 1-pinnatiséquées sessiles, à base inégalement cordée, pinnules des feuilles 2-pinnatiséquées à base décurrente .. Pteris

5' Pinnules sessiles ou subsessiles, suborbiculaires à oblongues-lancéolées, face dorsale glabre, à quelques écailles hyalines éparses ou à poils glanduleux.

Allosorus

\section{Pteris}

1 Feuilles en touffe lâche ; pétiole jaune, brun rougeâtre à la base ; limbe à contour largement ovale, 3-pinnatiséqué à la base, 2-pinnatiséqué au milieu, pinnatiséqué au sommet; segments primaires \pm opposés, ceux de la paire basale brièvement pétiolulés portant extérieurement 1-2 segments secondaires pinnatiséqués, les suivantes sessiles à base \pm décurrente, les supérieurs réduites à une pinnule et formant dans leur ensemble comme un segment terminal analogue aux latéraux; pinnules sessiles, décurrentes inférieurement, lancéolées subfalciformes, aiguës, serrulées; rachis portant à la base des nervures médianes des pinnules un prolongement sétiforme court souvent peu visible. Sores larges $0,75 \mathrm{~mm}$, ne dépassant guère le milieu de la pinnule. P. incompleta 
1' Feuilles en touffe assez dense ; pétiole brun; limbe lancéolé, atténué à la base et au sommet, pinnatiséqué ; segments primaires \pm opposés ou alternes très nombreux, espacés, linéaires, longuement atténués au sommet, aigus, inégalement cordés et sessiles à la base, serrulés sauf au niveau des sores ; segment terminal un peu décurrent d'un côté. Sores larges 1-1,5 mm, occupant parfois les $3 / 4$ et même les $4 / 5$ de la longueur du segment fertile. P. vittata

\section{Allosorus}

1 Rachis des feuilles couverts de poils glanduleux courts parfois en mélange avec d'autres plus longs. Limbe des feuilles à poils glanduleux courts sur la face dorsale A. tinaei

1' Rachis glabre à paléacé ....2

2 Pétiole glabre, pourpre noir, en général plus long que le limbe; rachis noir, glabre ou les secondaires portant quelques poils glanduleux; limbe deltoïde, 3-4-pinnatiséqué ; pinnules sessiles, entières ou pinnatilobées, vertes et glabres dessus, couvertes dessous de poils rubigineux, articulés, glanduleux...

A. hispanicus

2 Pétiole glabre ou couvert d'écailles, brun ou brunrouge, plus court que le limbe ou l'égalant; rachis glabre ou écailleux; limbe ovale, ovale-lancéolé, ovale-oblong, oblong-lancéolé, 2-3-pinnatiséqué ...3

3 Pétiole couvert d'écailles \pm espacées, à la fin glabrescent; segments primaires de limbe pétiolulés. Rhizome couvert d'écaille brunes.... A. pteridioides

3' Pétiole à écailles abondantes au moins à sa base ; face inférieure du limbe glabre ou à écailles hyalines. Rhizome couvert d'écailles ferrugineuses ..............4 4

4 Pseudo-indusie large de 0,5-1 mm, subcontinue, parfois lobée et à lobules sub-rectangulaires; pétioles à écailles abondantes seulement à sa base, rares dans la partie supérieure.

A. guanchicus

4' Pseudo-indusie généralement large et discontinue, à marges profondément fimbriée; pétiole abondamment couvert d'écailles sur toute sa longueur.

A. acrosticus

Salviniaceae: Au Maroc un seul genre Azolla

1 Face supérieure des feuilles flottantes à poils formés de 2(3) cellules (voir au microscope). Lobe supérieur des feuilles flottantes presque pointu, à marge membraneuses étroite. Pousses à croissance compacte et plate à la surface de l'eau ...A. cristata

1' Face supérieure des feuilles flottantes à poils unicellulaires (voir au microscope). Lobe supérieur des feuilles flottantes obtus, à marge membraneuses relativement large. Pousses à croissance plus lâche, les sommets des tiges croissant au dessus de la surface de l'eau

A. filiculoides

Selaginellaceae: Au Maroc, un seul genre Selaginella

1 Feuilles ovales-elliptiques, disposées en 4 rangées sur un même plan : les grandes en 2 rangées latérales, les petites en 2 rangées médianes............

S. denticulata

1' Feuilles étroitement linéaires, aristées au sommet, alternes en spirales, semblables.

S. balansae

\section{Thelypteridaceae}

1 Feuilles à limbe ovale-lancéolé, 2-pinnatifide; pétiole portant à la base des écailles brunes caduques et des poils blancs longs et courts; rachis couvert de poils blancs longs et courts... Cyclosorus dentatus

1' Feuilles à limbe lancéolé à oblong-lancéolé, 2-pinnatiséqué; pétiole et rachis glabres Thelypteris

2 Face inférieure du limbe jeune portant des poils et des glandes jaunâtres. Limbe 2-penné. Pinnules oblongues, entières ou faiblement denticulées, les fertiles à la fin à marge révolutée, devenant \pm deltoïdes

T. palustris

2' Face inférieure du limbe portant des poils, parfois absents, des écailles et des glandes sessile brillantes, rouges à oranges. Limbe 1-penné; pennes pinnatipartites à pinnatifides

T. interrupta

\section{Etats de présence et de conservation}

A l'instar des autres pays du Sud et de l'Est de la Méditerranée, le Maroc connait sur presque l'ensemble de son territoire une très forte pression anthropozoogène sur la biodiversité : prélèvement de bois, défrichement, parcours, surexploitation des plantes aromatiques et médicinales... Concernant les Ptéridophytes, leur importance socio-économique étant presque insignifiante au niveau national, on peut dire que, dans l'ensemble et jusqu'à présent, elles ne font pas l'objet de menaces spécifiques directes, exceptions faites pour quelques espèces parfois exploitées pour des usages en médecine traditionnelle, comme : Adiantum capillus-veneris, Asplenium ceterach, Asplenium scolopendrium, Dryopteris filixmas, Equisetum ramosissimum, Polypodium cambricum, Pteridium aquilinum et Selaginella balansae (Fennane \& Rejdali 2016). Cependant, ce constat ne veut nullement dire que les fougères se portent bien au Maroc. Au contraire, elles sont certainement en régression. II y a absence totale de données quantitatives concrètes à ce sujet, mais la tendance générale à la régression ne fait pas de doute, vu la grande vulnérabilité et la faible capacité de résilience des biotopes humides qui sont les lieux exigés par la plupart des espèces. La sécheresse et la dégradation par l'homme agissent directement et rapidement sur ces biotopes, et malheureusement ces deux facteurs prennent de plus en plus d'ampleur ces dernières décennies.

Dans un récent travail, nous avons estimé à 16 le nombre d'espèces menacées (catégories CR, EN et VU de I'IUCN) (Fennane 2016). Elles sont toutes rares, ou très rares, d'après les informations disponibles ; d'autres, peu connues, le seraient aussi. Dans ce qui suit, nous reprenons ce travail pour dresser un état des lieux sur les états de 
présence et de conservation des fougères au Maroc.

Espèces communes, à large répartition géographique

Les connaissances actuelles sur les fougères du Maroc laissent prétendre que la moitié des espèces sont plus ou moins communes, sans menaces directes et effectives à court et moyen termes. Elles pourraient ainsi être classées dans la Catégorie LC («Least Concern» = Préoccupation mineure) de la Liste Rouge de I'UICN (Union Mondiale pour la Nature). Ces espèces sont les suivantes ; elles appartiennent à 12 familles différentes :

\section{Aspleniaceae}

Asplenium adiantum-nigrum $\mathrm{L}$.

Asplenium ceterach $\mathrm{L}$.

Asplenium obovatum Viv.

Asplenium petrarchae (Guérin) DC.

Asplenium ruta-muraria L.

Asplenium scolopendrium $\mathrm{L}$.

Asplenium trichomanes $\mathrm{L}$.

Asplenium viride Hudson

Athyriaceae

Athyrium filix-femina (L.) Roth

Cystopteridaceae

Cystopteris fragilis (L.) Bernh.

Dennstaedtiaceae

Pteridium aquilinum (L.) Kuhn Equisetaceae

Equisetum ramosissimum Desf.

Equisetum telmateia Ehrh.

Isoëtaceae

Isoëtes histrix Bory

Isoëtes velata A. Braun

Marsileaceae

Marsilea strigosa Willd.

Ophioglossaceae

Ophioglossum lusitanicum L.

Ophioglossum vulgatum L.

Osmundaceae

Osmunda regalis L.

Polypodiaceae

Polypodium cambricum L.

Peridaceae

Adiantum capillus-veneris L.

Allosorus acrosticus (Balb.) Christenh.

Allosorus guanchicus (Bolle) Christenh.

Allosorus hispanicus (Mett.) Christenh.

Allosorus pteridioides (Reich.) Christenh.

Anogramma leptophylla (L.) Link

Cosentinia vellea (Aiton) Tod.

Selaginellaceae

Selaginella balansae (A. Braun) Heiron.

Selaginella denticulata (L.) Spring.

Cette fraction de la flore cryptogamique commune se rencontre dans toutes les divisions géographiques du pays et sur l'ensemble des tranches altitudinales depuis les basses plaines jusqu'aux hautes montagnes. La plupart ont une large distribution mondiale, au moins méditerranéenne ; la seule exception est Selaginella balansae, endémique nationale.

\section{Espèces rares ou menacées}

Le cortège des fougères rares ou menacées au Maroc compte au moins 16 espèces qui pourraient être classées dans l'une des Catégories suivantes de la Liste rouge de l'IUCN : CR («Critically endangered» = En danger critique), EN (Endangered = En danger), VU (Vulnérable). Nous les présentons ci-après, avec un commentaire plus ou moins détaillé, extrait de notre travail Fennane (2016). Pour les abréviations des divisions géographiques, voir fig. 3.

Asplenium aethiopicum (Burm. f.) Becherer

AA-2 (jbel Imzi et Adad Medni au NNE d'Anezi) [Benabid \& Cuzin 1997]. Falaises quartzitiques très difficile d'accès, ce qui laisse l'espèce à l'abri de la dégradation anthropique. Les menaces probables proviendraient de l'aridification du climat. La zone d'occurence fait partie de la Réserve de biosphère «Arganeraie» et du Site d'Intérêt Ecologique et Biologique d'Anezi, mais aucune mesure de conservation particulière n'existe sur le terrain.

\section{Asplenium marinum $\mathrm{L}$.}

Mam-2 (Cap Meddouza) Man-3 (Temara) LM (Melilia ; Gourougou) R-1 (Cap Spartel ; grottes d'Hercule). Falaises maritimes et sublittorales. Les informations sur la répartition géographique de cette espèce au Maroc sont très anciennes. Sachant que le littoral marocain est de plus en plus anthropisé, il y a de quoi être inquiet sur le sort de l'espèce, qui jusqu'à preuve du contraire doit être considérée en danger.

\section{Asplenium sagittatum (DC.) Bange}

R (Bni Hosmar, Chaouène, Uad-el-Kannar [Mateos \& Valdés 2009]) LM (piste Ahfir-Aïn Almou) [Molero \& Montserrat 2006]. Espèce rare, à aire très probablement en régression sous l'effet des sécheresses récurrentes, vu la nature particulière des habitats qui sont des rochers humides ombragés.

\section{Asplenium septentrionale (L.) Hoffm.}

Les informations sur la répartition de cette espèce au Maroc proviennent du Catalogue des plantes du Maroc (Jahandiez \& Maire 1932:5 et Emberger \& Maire 1941:917) : HA sur rochers porphyriques (Rherhaya, vallée de l'açif Ouenkrim et adrar-n-Ouaraout ; Ourika) MA-3 (rochers 
basaltiques dans les avens du plateau au dessus d'Azrou) R-2 (Tidighine). Depuis 1941, aucune information nouvelle n'a été publiée, même pas dans le Rif où les explorations floristiques ont été relativement importantes au cours des deux dernières décennies.

\section{Dryopteris filis-mas (L.) Schott}

HA-3 (Adrar-n-Ouaraout ; Erdouz) R-1 (au NW de Tetouan) [Ennabili \& Gharnit 2003] R-2 (j. Ighermalez ; Tidighine et monts des Ketama). Maire (1952) donne cette espèce très rare, et il est certain que la tendance générale pour ses habitats (ravins humides, ruisselets, humus forestier, fissures ombreuses des rochers) est à la régression ; la citation de Ennabili \& Gharnit (2003) est la seule enregistrée depuis les années 1950.

\section{Polystichum aculeatum (L.) Roth}

Présence rare sur des sommets du HA (Rherhaya; Glaoua; Ourika) et MA central (jbel Hayyan) ; signalé également dans le Rif, probablement par confusion avec Polystichum setiferum.

\section{Isoëtes durieui Bory}

Espèce signalée au Maroc depuis longtemps, mais avec doute ; présence récemment confirmée dans le Rif occidental par Valdés et al. (2002) et Mateos \& Valdés (2010).

\section{Isoëtes longissima Bory}

Connue dans deux localités seulement: MA-3 (près d'Ito) (Maire 1952), subsp. intermedia (Trab.) Troìa \& Greuter ; Op-2 ( $\mathrm{N}$ de Boumia) (Dobignard 2002), subsp. adspersa (A. Braun) Troìa \& Greuter. Le genre Isoëtes est peu étudié au Maroc, et il n'est pas exclu que d'autres localités pourraient être découvertes dans le futur. Dans tous les cas, l'espèce reste très menacée vu ses habitats (micro-lacs) fragiles, soumis à une forte pression anthropozoogène sous des conditions climatiques de plus en plus sèches.

\section{Isoëtes setacea Lam.}

Mam-4 (Haouz ?) Man-3 (environs de Sidi Bettache; Maâmora?). Les informations sur la répartition de cette espèce au Maroc sont résumées et analysées par Titolet \& El Oualidi (2000) et par Dobignard (2002) ; on en retient une présence confirmée dans la région Benslimane / Sidi Bettache et douteuse ailleurs dans le Haouz et le Gharb. Partout, ses habitats, réels ou potentiels (dayas temporaires ou permanents), subissent une très forte pression anthropique : surpâturage, drainage et pompage de l'eau, urbanisation, rejets de déchets domes-tiques et industriels...

\section{Isoëtes tiguliana Gennari}

Man-3 (dayas aux environs de Benslimane et de Salé) (Maire 1952), mais pour Dobignard (2002), il sagirait plutôt de I. setacea; présent également au Tazekka (Valdés et al. 2002) et à Bab Taza dans le Rif occidental (Maire 1931) et (Mateos \& Valdés 2009). Espèce très localisée, de milieux spécialisés (microlacs) très fragiles; seule la station du Rif est certaine, récemment confirmée (Mateos \& Valdés ibid.)

\section{Marsilea minuta L.}

Une seule citation au Maroc: Man-2 (basse vallée du Loukkos) (Mathez 1980a); la station signalée appartient à un ensemble de marais qui subissent une pression anthropique constamment croissante sous l'effet du drainage, de la modernisation du système agricole et de l'urbanisation.

\section{Pilularia minuta Durieu}

Pour Fennane \& Ibn Tattou (1998), cette espèce est très rare ; pendant plus de 50 années, elle était connue de 3 localités seulement: Tiflète, Rommani et Benslimane. En 2002, Valdés et al. la citent à «Tanger, W Rif, Zerhoun» sans détails. Plus tard, toujours au Maroc septenrional, des localités précises sont signalées (Mateos \& Valdés 2009; Chambouleyron 2012). L'espèce reste rare, et surtout menacée, eu égard à ses habitats (dayas) très perturbés et affectés par le manque d'eau de plus en plus réel à l'échelle de l'ensemble du pays.

\section{Ophioglossum polyphyllum A. Braun}

Anciennement citée dans Ms-3 (basse vallée de I'o. Noun ; El Aïoun du Drâa). Présence récemment confirmée dans la région d'El Aïoun du Drâa (Garcin 2016).

\section{Polypodium vulgare $\mathrm{L}$.}

Espèce signalée il y'a près d'un siècle sur le revers nord du massif du Toubkal ; une deuxième localité a été découverte dans le Rif (Kaâ Asrass) en 2003 par Mateos \& Valdés. Selon Med-Checklist (Greuter et al. 1984), P. vulgare (s.l.) inclut trois espèces : P. cambricum L., P. interjectum Shivas et $P$. vulgare L.. La présence de l'espèce (s.s.) au Maroc est confirmée par la station du Rif ; celle du Toubkal mérite d'être vérifiée. Eu égard aux confusions possibles avec $P$. cambricum, il n'est pas impossible que $P$. vulgare $L$. existe ailleurs au Maroc.

\section{Pteris vittata $\mathrm{L}$.}

Signalée pour la première fois à l'état naturel par Sefanesco (1968) entre Tétouan et Sebta et entre Bouhamed et Jebha ; la première localité a été confirmée par Mathez (1980b). Avant ces dates, la plante était connue dès 1952 à Skhirate (SW 
de Rabat) (Gattefossé 1952), mais probablement introduite. En 1997, elle a été rencontrée par Dobignard à Barga (SW de Larache). Récemment, après l'année 2000, d'autres localités ont été découvertes dans le Rif occidental (Dobignard 2002, 2009 ; Chambouleyron 2012). Dans l'ensemble, il s'agit de populations réduites, catonnées dans des micro-habitats humides (ruisseaux, rochers humides...).

\section{Thelipteris palustris Schott}

Citée par Jahandiez \& Maire $(1931,1934)$ dans la région de Larache (marais de Bou-Charen), sous le nom Dryopteris thelyptris (L.) A. Gray. En 1970, l'espèce est signalée par Mathez \& Sauvage non loin de la ville, en allant vers Açilah. Au vu de ces données, et sachant que les zones humides de la région de Larache sont soumises à de fortes pressions anthropiques (parcours, urbanisation, pollution, drainage...), il y a à craindre que l'espèce soit très menacée si elle est encore présente.

\section{Espèces insuffisamment connues}

Selon nos investigations, le nombre de fougères peu, ou très peu connues au Maroc, s'élève à 22 espèces pour lesquelles le doute est plus ou moins fort sur leur présence, leur abondance ou bien sur leur statut (indigène ou exotique). Elles appartiennent à 10 familles différentes, dont les Blechnaceae, Davalliaceae et Salviniaceae. Ces trois familles méritent une attention particulière ; chacune est représentée chez nous, par un seul genre et une ou deux espèces, toutes mal connues.

Toutes les espèces concernées sont listées ciaprès, avec des commentaires empruntés à notre travail, Fennane (2016).

\section{Asplenium anceps Hook. \& Grev.}

La présence et la valeur taxonomique de cette fougère restent à élucider au Maroc. Maire (1952) cite Asplenium trichomanes var. anceps sur le mont Hadid et dans le Grand Atlas, et jusqu'à présent, nous n'avons pas plus d'informations.

\section{Asplenium fontanum (L.) Bernh.}

Maire (1959) signale cette espèce sur les rochers du HA-4 (Rhat, Mgoun, Aïoui) entre 2600 et $3500 \mathrm{~m}$ d'altitude. Depuis, on ne connait pas plus ; ces mêmes informations sont reprises dans la littérature par beaucoup d'auteurs sans précisions, ni détails supplémentaires.

\section{Asplenium foreziense Magnier}

Signalée au Maroc par Valdés et al. (2002) dans le Nord du pays, sans localités précises. Dobignard \& Chatelain (2010) la donnent présente au Maroc et en Algérie, mais cette présence est mise en doute par les mêmes auteurs dans la Base de données des plantes d'Afrique [PI_Afr] ! L>origine de l,information semble être Maire (1952) qui cite Asplenium obovatum var. numidicum en Algérie et au Maroc (Rif : Bni Hdifa et j. Outka), tout en faisant le rapprochement entre cette variété et Asplenium lanceolatum subsp. foresiacum (Legrand) Mattir. (synonyme de $A$. foreziense selon [EMBase]). A la lumière de ces seules informations disponibles, il est permis d'avoir des doutes sur la présence et la valeur taxonomique de cette fougère au Maroc.

\section{Asplenium hemionitis $\mathrm{L}$.}

Fennane \& Ibn Tattou (1998) donnent cette espèce rare dans l'aire suivante : Man-3 (oueds Cherrat, Nefifikh, Akrech et N'kheila) LM-1 (Melilia) R-1 (j. Kebir). Depuis, de nouvelles localités ont été signalées dans le Rif occidental et central [Ennabili \& Gharnit 2003] [Mateos \& Valdés 2009]. L'aire d'occupation de l'espèce reste mal connue, de même que les tendances de ses populations.

Asplenium hispanicum (Coss.) Greuter \& Burdet

Espèce présente dans au moins 5 divisions géographiques du Maroc : HA-4 (Ifrane près d'Azilal) MA-3 (Sefrou, Kebab, sources Oum-erRbiâ, entre Azrou et Ain Leuh) Man-1 (Zerhoun) Op-1 (région d'Ain Zorah) R-2 (Bab Rouida ; Talassemtane). L'espèce est calcicole, sciaphyle, se cantonne dans les fissures et poches terreuses des rochers entre environ 1000 et 1500 m. Elle ne semble pas être vraiment rare, mais ses habitats fragiles poussent à surveiller de près son évolution.

\section{Asplenium seelosii Leybold}

Maire (1928) est l'auteur de la première annonce de cette espèce au Maroc : MA-2 (Lalla Oum el-Bent au Tichchoukt, fissures des rochers calcaires). Une $2^{\mathrm{im} e}$ citation, dans le Haut Atlas cette fois, revient à Quézel (1951) : HA-4 (rochers calcaires, ça et là dans tout le massif du Rhat au dessus de 3000 m). Fennane \& Ibn Tattou (2005) et Valdès et al. (2002) la donnent respectivement au Moyen Atlas oriental (Bou Nacer) et au Rif central, mais sans références bibliographiques précises.

\section{Blechnum spicant (L.) Roth}

Jusqu'au début des années 1950, l'espèce était connue uniquement dans 4 localités : R-1-2 (jbel Kebir ; j. Outka ; j. Bou-Hachem ; cédraie de Bab Besen). Récemment, Ennabili \& Gharnit (2003) et Chambouleyron (2012) ont publié de nouvelles stations, toujours dans cette même région, réparties sur les jbels Sougna, Bou-Hachem, Âlam et Khesana. L'espèce ne semble pas être vraiment 
rare, mais ses habitats sont fragiles (ravins humides et bords de ruisselets), très sensibles au manque de pluies.

\section{Davallia canariensis (L.) Sm.}

Ms-3 (khneg Lehmam) AA-2 (j. Imzi et Adad Medni au NNE d'Anezi) LM R-1-2. Rochers gréseux et troncs d'arbres des zones littorales et sublittorales. L'aire très disloquée de l'espèce et ses habitats soumis à une pression anthropique de plus en plus forte laissent supposer une tendance à sa régression.

\section{Dryopteris affinis (Lowe) Fraser-Jenkins}

Espèce signalée pour la 1ère fois au Maroc en 1943 par Sauvage sous le nom Dryopteris filixmas : MA-4 (oueds permanents dans la subéraie de Bab Azhar). En 1954, ce même auteur la reclasse sous Dryopteris paleacea (Sw.) HandMazz. (synonyme de $D$. borrerı). En fait, cette espèce semble plutôt être $D$. affinis selon Greuter (1980). Fraser-Jenkins (1980) la signale au Maroc, mais sans localité précise, ni citation de spécimen d'herbier témoin. Présence confirmée par Carine et al. (2006) : Rif occidental (j. Bou-Hachem). Dans tous les cas, il s'agirait de localités en limite d'aire, uniques au Maroc et en Afrique.

\section{Dryopteris borreri (Newman) Oberh. \& Tavel}

Présence douteuse au Maroc (Greuter 1980; EMBase); seul Dobignard \& Chatelain (2010) affirment sa présence, mais sans argument.

\section{Polystichum lonchitis (L.) Roth}

Une seule localité connue pour le Maroc : HA-4 (j. Rhat) (Quézel 1951) ; rien ne prouve que l'espèce est toujours présente. Dans tous les cas, il s'agit d'une station relictuelle, en limite d'aire, unique en Afrique.

\section{Polystichum setiferum (Forssk.) Woyn.}

Cette espèce est indiquée dans le Moyen Atlas et le Rif, mais sans détails sur l'aire de répartition. Les seules informations, un peu précises, sont données par Sauvage (1943) dans le Tazekka (oueds permanents de la forêt de Bab Azhar) et parhel

Mateos \& Valdés (2009) et Valdés (2013), quoique pour ces dernières il y a une confusion probable avec $P$. aculeatum.

\section{Equisetum arvense $\mathrm{L}$.}

Citée par Sennen \& Mauricio (1933) dans la région de Mélilia, mais pour Maire (1952), il s'agit d'une confusion avec E. ramosissimum. Présence jamais confirmée au Maroc.
Botrychium lunaria (L.) Swatrz

HA R. Les connaissances sur cette espèce au Maroc n'ont pas évolué depuis plus d'un demi siècle ; ce sont celles du Catalogue des plantes du Maroc (Jahandiez \& Maire 1931 ; Emberger \& Maire 1941), reprises par la Flore de l'Afrique du Nord (Maire 1952). Quézel (1957) a apporté quelques détails en indiquant l'espèce dans 19 de ses relevés floristiques répartis entre les massifs de Tichka, Toubkal, Oukaimeden et Mgoun. Pour la population du Rif, au jbel Tidighin, aucune donnée récente n'apporte des précisions utiles malgré le nombre important de publications floristiques sur le Rif ces deux dernières décennies. Ecologiquement, l'espèce est présente sur les hauts sommets (2000-3500 m), où elle est liée à des pozzines, essentiellement sur substrats siliceux ; ces milieux très particuliers seraient en voie de régression, par insuffisance de pluies et de neige.

\section{Polypodium interjectum Shivas}

La seule citation au Maroc revient à Salvo et al. (1992) : R (Khemis Bni Arouss; Bab Besen). L'espèce fait partie de l'aggrégat Polypodium vulgare différemment interprété au niveau spécifique (s.s.) suivant les auteurs. Des confusions sont toujours possibles ; d'ailleurs, la présence de $P$. interjectum au Maroc est mise en doute par Dobignard \& Chatelain (2010) et (EMBase).

Allosorus tinaei (Tod.) Christenh.

Signalée au Maroc, sous le nom Cheilanthes duriensis Mendonça \& Vasc., par Saenz de Rivas \& Rivas-Martinez (1979), sans précision de localité, ni de spécimen d'herbier. Sans preuves concrètes, la présence de cette espèce chez nous reste à confirmer.

\section{Paragymnopteris marantae (L.) K.H. Shing}

Une seule localité connue au Maroc : R-2 (o. Damazine à l'est de Bou Hmed) (Sefanesco 1966); aucun autre auteur n'a confirmé sa présence. L'extinction de l'espèce à l'échelle régionale n'est pas impossible après que l'autre unique station d'Algérie n'a plus été retrouvée (Sefanesco 1966).

\section{Pteris incompleta Cav.}

La seule région au Maroc (Tangérois : jbel Kebir; Cap Spartel) où l'espèce a été signalée, est soumise à de très fortes pressions ces dernières décennies (urbanisation, déforestation...) ; il y a à craindre qu'elle n'y soit plus présente (Sefanesco 1968).

\section{Azolla cristata Kaulf.}

Présence douteuse au Maroc. 
Azolla filiculoides Lam.

Espèce non indigène ; naturalisée.

Cyclosorus dentatus (Forssk.) Ching

Présence douteuse au Maroc.

Thelypteris interrupta (Willd.) K. Iwats.

Signalé au Maroc, pour la 1ère fois, par Jahandiez \& Maire (1931) dans la région de Larache au nord de Lalla Mimouna, sous le nom Dryopteris gongylodes susbp. propinqua (R.Br.) C.Chr.. Valdés et al. (2002) l'ignorent en considérant qu'il s'agit d'une confusion avec Thelypteris palustris Schott., avis semble-t-il partagé par Dobignard \& Chatelain (2010) qui mettent en doute la présence de l'espèce au Maroc.

\section{Conclusion}

La richesse, la diversité et les menaces concernant la flore ptéridophytique nationale sont dans l'ensemble méconnues. Les recherches systématiques et floristiques sur cette flore, un peu particulière, sont faibles, ce qui nourrit l'indifférence du grand public et des gestionnaires à son égard.

La valorisation, la protection et la conservation de notre patrimoine ptéridophytique ne peuvent pas réussir dans de telles conditions. II y a encore beaucoup de travail à faire pour amener à un niveau raisonnable les connaissances sur ce patrimoine, notamment des points de vue constituants (familles, genres, espèces...), habitats et aires de répartition.

Selon l'état des lieux dressé dans le présent travail, il y a urgence pour entreprendre des actions de protection de certaines espèces sérieusement menacées. Ces opérations seraient possibles et envisageables au moins pour des localités cibles situées à l'intérieur des aires protégées actuelles: parcs, réserves...

Ce travail a été également l'occasion pour produire une nouvelle clé d'identification des fougères du Maroc, intégrant les progrès scientifiques enregistrés sur ce groupe depuis la publication de celle de Salvo \& Ouyahya en 1999 dans la Flore pratique du Maroc.

\section{Références}

Benabid A. \& Cuzin F. (1997). Populations de dragonnier (Dracaena draco L. subsp. ajgal) au Maroc: valeurs taxinomique, biogéographique et phytosociologique. C. R. Acad. Sci. Paris, Sci. de la vie 320: 267-277.

Carine M.A., Rumsey F.J., Ait-Lafkih et al. (2006). New plant collections from the North Morocco checklist area. Lagascalia 26: 196-218.

Chambouleyron M. 2012. Contribution à la connaissance de la flore de la Péninsule Tingitane (Maroc). Lagascalia
32: 35-227.

Dobignard A. \& Chatelain C. (2010). Index synonymique de la flore d'Afrique du Nord. Volume 1. Edit. Conserv. Jardin Bot. de Genève.

Dobignard A. 1997. Nouvelles observations sur la flore du Maroc. 3. Candollea 52: 119-157.

Dobignard A. (2002). Contributions à la connaissance de la flore du Maroc et de l'Afrique du Nord. Nouvelle série I. J. Bot. Soc. Bot. France 20: 5-43.

Dobignard A. (2009). Contributions à la connaissance de la flore du Maroc et de l'Afrique du Nord, Nouvelle série 2. La flore du Nord-Maroc. J. Bot. Soc. Bot. France 46-47: 3-136.

EMBase (accés, avril 2017). Base de données EuroMed Plant Base. http://ww2.bgbm.org/EuroPlusMed/query. asp

Emberger L. \& Maire R. (1941). Catalogue des plantes du Maroc. Tome 4. Minerva, Alger.

Ennabili A. \& Gharnit N. (2003). Checklist and diversity of wetland flora (Pteridophyta and Spermatophyta) from the Mediterranean Morocco. Lagascalia 23: 7-25.

Fennane M. \& Ibn Tattou M. (2005). Flore vasculaire du Maroc : Inventaire et chorologie. Vol. 1. Trav. Inst. Sci., Rabat, Sér. Botanique n 37. 483 p.

Fennane M. \& Ibn Tatto M. (2012). Statistiques et commentaires sur l'inventaire actuel de la flore vasculaire du Maroc. Bull. Inst. Sci., Série Sc. Vie, 34: 1-9. Rabat.

Fennane M. \& M. Rejdali (2016). Aromatic and medicinal plants of Morocco: Richness, diversity and threats. Bull. Inst. Sci., Rabat, Sci. Vie, 38: 13-28.

Fennane M. (2016). Eléments pour un Livre rouge de la flore vasculaire du Maroc. Fasc. 1: Pteridophyta (version 1). Edit. Tela-Botanica, $16 \mathrm{p}$.

Fraser-Jenkins C.R. (1980). Dryopteris affinis: a new treatment for a complex species in the European Pteridophyte flora. Willdenowia 10: 107-115.

Garcin A. (2016). La vallée inférieure de l'oued Drâa: Joyau phytogéographique et floristique du Sud-Ouest marocain. Bull. Inst. Sci., Rabat, Sc. Vie 38: 1-12.

Gattefossé J, (1952). Contribution à la connaissance de la flore du Maroc. Fasc. III. Bull. Soc. Sc. Nat. Maroc 32: 53-59.

Greuter W. (ed.), (1980). Med-Checklist Notulae 1. Willdenowia 10: 13-21.

Greuter W., Burdet H.M. \& Long G., (eds). (1984). MedChecklist : Inventaire critique des plantes vasculaires des pays circum-méditerranéens. Vol. 1. Edit. Conserv. et Jard. Bot. de Genève.

IUCN (Union Mondiale pour la Naure). Site web RedList (accès, avril 2017). http://www.iucnredlist.org

Jahandiez E. \& Maire R. (1931). Catalogue des plantes du Maroc. Tome 1. Imprimerie Minerva, Alger.

Maire R., (1928). Contributions à l'étude de la flore de l'Afrique du Nord. Fasc. 13. Bull. Soc. Sc. Nat. Maroc 8: 128-143.

Mateos M.A. \& Valdés B. (2003). Nuevas especies para el N de Marruecos I. Lagascalia 23: 173-176.

Mateos M.A. \& Valdés B., (2009). Catálogo de la flora vascular del Rif occidental calizo (N de Marruecos). I. Selaginellaceae - Rosaceae. Lagascalia 29: 105-257.

Mathez J. (1980a). Marsilea minuta L. In Greuter (ed.). 
Med-Checklist Notulae 1. Willdenowia 10: 13-21.

Mathez J. (1980b). Pteris vittata L. In Greuter (ed.). MedChecklist Notulae 2. Willdenowia 10: 227-232.

PI_Afr. (accès, avril 2017). Base de données des Plantes d'Afrique. http://www.ville-ge.ch/musinfo/bd/cjb/africa/ index.php?

PI_List (accès, avril 2017). The Plant List : A working list of all plant species. http://www.theplantlist.org/

PPGI, (2016). A community-derived classification for extant lycophytes and ferns. J. Syst. Evol. 54(6) : 563603.

Quézel P. (1951). Contribution à la flore du Grand Atlas oriental. Bull. Soc. Sc. Nat. Maroc 31: 253-264.

Quézel P. (1957). Peuplement végétal des hautes montagnes de l'Afrique du Nord. Lechevalier, Paris, $463 \mathrm{p}$.

Saenz de Rivas C. \& Rivas-Martinez S., (1979). Revision del genero Cheilanthes en Espana. Lagascalia 8(2): 215-241.

Salvo A.E. \& Ouyahya A. (1999). Pteridophyta. In Fennane M., Ibn Tattou M., Mathez J., Ouyahya A. \& El Oualidi J. (eds.). Flore Pratique du Maroc. Vol. 1. Trav. Inst. Sci. Rabat, Sér. Bot. 36: 28-56.

Salvo A.E., Márquez A.L., Pérez Latorre A., Nieto Caldera J.M. \& Cabezudo B. (1992). Contribución a la flora pteridofítica de Marruecos 23. Acta Bot. Malacitana
17: 287-290.

Sauvage Ch. (1943). Annotations au Catalogue des plantes du Maroc. Fasc. 1. Bull. Soc. Sc. Nat. Maroc 23: $127-140$.

Sauvage Ch. (1954). Principaux résultats de l'excursion marocaine du VIIlème Congrès international de botanique. C. R. Soc. Sc. Nat. Maroc, tome 20. Comptes rendus 1954, n 8: 194-195.

Sennen F. \& Mauricio (1933). Catálogo de la flora del Rif Oriental. Gráficas la Ibérica Marina, Melilia, 159 p.

Stefanesco E. (1966). Espèces nouvelles pour la flore du Maroc: Sphagnum amblyphyllum Russ. et Notholaena marantae (L.) R. Br. Al Awamia 21: 125-126. INRA, Rabat.

Stefanesco E. (1968). Espèce nouvelle pour la flore marocaine : Pteris longifolia L. Al Awamia 27: 140. INRA, Rabat.

Titolet D. \& El Oualidi J. (2000). Isoetes setacea Lam. / I. velata subsp. adspersa (A. Braun) Batt. \& Trabut. Acta Bot. Malacitana 25: 252-253.

Valdés B. (2013). Checklist of the vascular plants collected during the fifth «lter Mediterraneum» in Morocco, 8-27 june 1992. Bocconea 26: 13-132.

Valdés B., Rejdali M., Achhal A. et al. (eds), (2002). Catalogue des plantes vasculaires du Nord du Maroc, incluant clés d'identification. Vol. 1. CSIC. Madrid. 